\title{
Influência da equoterapia no desenvolvimento psicomotor de pessoas com necessidades especiais
}

Gardenia de Oliveira Barbosa*

Mey de Abreu van Munster**

\section{Resumo}

A equoterapia é um processo terapêutico e educacional que utiliza o cavalo com a finalidade de proporcionar ganhos físicos e psíquicos para pessoas com necessidades especiais. Nesse estudo objetivou-se analisar a influência da equoterapia no desenvolvimento psicomotor de pessoas com necessidades especiais. Para isso, foi realizada uma pesquisa bibliográfica e a partir dos artigos encontrados procedeu-se a análise por meio da leitura dos resumos e seleção dos que envolviam os aspectos psicomotores relacionados à intervenção equoterapêutica. Como resultados, foram encontrados 15 artigos relevantes ao objeto de estudo em questão, os quais apontaram evidências quanto a ganhos em habilidades motoras, devido à intervenção com a utilização do cavalo. Foi possível analisar a periodicidade de publicação dos artigos, identificar as populações beneficiadas pela prática da equoterapia e as principais teorias que oferecem sustentação às pesquisas. Por meio da revisão de literatura, concluiu-se que a equoterapia pode ser uma ferramenta eficaz como intervenção nos aspectos psicomotores de pessoas com necessidades especiais.

Palavras-chaves: Equoterapia; Psicomotricidade; Necessidades especiais.

\footnotetext{
* Mestranda do Programa de Pós-Graduação em Educação Especial/Universidade Federal de São Carlos (UFSCar), São Carlos São Paulo.

* Professora Doutora do Programa de Pós-Graduação em Educação Especial/Universidade Federal de São Carlos (UFSCar), São Carlos São Paulo.
} 


\title{
Influence of hippotherapy on psychomotor development of people with special needs
}

\begin{abstract}
Hippotherapy is a therapeutic and educational process that uses a horse with the purpose of providing physical and psychological improvement for people with special needs. In this study, the goal was to analyze the influence of hippotherapy on psychomotor development of people with special needs. In order to do this, an extensive bibliographical research was performed and from the items found an analysis was carried out through reading the abstracts and selecting the works which involved psychomotor aspects related to hippotherapeutic intervention. As a result, 15 articles were found relevant to the object of this study, which showed evidence for gains in motor skills due to the intervention with the use of horses. It was possible to analyze the frequency of publication of articles on the area, identify the population who benefited from the practice of hippotherapy and the main theories that offer support to research. Through careful literature review, it was possible to conclude that hippotherapy can be an effective tool as intervention in the psychomotor aspects of people with special needs.
\end{abstract}

Keywords: Hippotherapy; Psychomotor; Special needs.

\section{Introdução}

A equoterapia é um processo terapêutico e educacional que utiliza o cavalo como meio, a fim de proporcionar ganhos físicos e psíquicos para pessoas com necessidades especiais (WICKERT, 1995; ANDE-BRASIL; 2011).

Os ganhos físicos estão relacionados ao movimento tridimensional realizado pelo cavalo ao passo, caracterizado por ser uma andadura ${ }^{1}$ rolada ou marchada, simétrica ${ }^{2}$ e ritmada ${ }^{3}$ (WICKERT, 1995).

O movimento tridimensional contínuo, associado à torção pélvica da ordem de oito graus para a direita e para a esquerda em um único passo do cavalo, proporciona ao praticante de equoterapia em montaria, aferências motoras que são transmitidas ao sistema nervoso central (SNC) gerando respostas que ativam todo o organismo (WICKERT, 1995). 
Sendo assim, a equoterapia por meio do movimento tridimensional realizado pelo cavalo proporciona inputs sensório-motores que influenciam adequadamente no processamento sensorial e neuromotor, refletindo no equilíbrio, postura, força e coordenação sensório-motora; pois ocorrem inúmeros estímulos ao sistema vestibular e proprioceptivo, devido ao constante deslocamento do centro de gravidade, dissociação escapular e pélvica, mudanças no campo visual, estímulos táteis e olfativos (BENDA; McGIBBON; GRANT, 2003; MEREGILLANO, 2004).

O cavalo atua como instrumento cinesioterapêutico muito valioso, devido às inúmeras oportunidades de ações que oferece ao praticante sobre seu dorso, tais como: mudanças no ritmo e direção do cavalo, movimentos de aceleração e desaceleração do passo, alterações de postura sobre o cavalo, transição de postura com o cavalo em movimento, diferentes posicionamentos dos estribos ou a não utilização dos mesmos, entre outros (MEREGILLANO, 2004).

\section{Objetivo}

O estudo tem por objetivo analisar a influência da equoterapia no desenvolvimento psicomotor de pessoas com necessidades especiais.

\section{Método}

Foi realizada uma vasta pesquisa bibliográfica que compreendeu quatro fases: a identificação (reconhecimento do assunto pertinente ao tema), localização (busca em bibliotecas ou fontes on-line), compilação (reunião sistemática das informações) e fichamento (transcrição dos dados em fichas com exatidão e cuidado) (MARCONI e LAKATOS, 1990); sem limite de data, nas bases de dados Web of Science, Biblioteca Virtual em Saúde (BVS), Scopus, Medline e no site da ANDE-Brasil, utilizando a palavrachave "hippotherapy" e os termos sinônimos apontados pelo Descritores de Ciências da Saúde (DECS) que são "equine assisted psycotherapy", "horseback riding therapy" e "recreation horseback riding therapy". A partir dos artigos encontrados procedeu-se a análise por meio da leitura dos resumos e seleção daqueles envolvendo os aspectos psicomotores relacionados à intervenção equoterapêutica.

\section{Resultados}

Foram encontrados 159 artigos, abordando inúmeros assuntos referentes à equoterapia, tais como menção a equipamentos de simulação 
do movimento tridimensional do cavalo, teste de equipamentos como os de captação de pressão quando praticante em montaria, comentários históricos, artigos de opinião, sugestão da equoterapia como prática terapêutica para diversos acometimentos, angulação dos membros inferiores conforme o posicionamento dos estribos, influência da equoterapia nas habilidades motoras, entre outros. No entanto, os que faziam referência a ganhos nas habilidades motoras com utilização do cavalo, de modo a relacionar-se com a psicomotricidade e que possibilitassem a construção do conhecimento em questão foram 15 artigos. Os artigos que apontaram evidências quanto a ganhos em habilidades motoras devido à intervenção com a utilização do cavalo encontram-se descritos no quadro a seguir.

Quadro 1. Artigos relacionados ao ganho nas habilidades motoras

\begin{tabular}{|l|l|l|l|}
\hline Ano & \multicolumn{1}{|c|}{ Autor } & \multicolumn{1}{|c|}{ Título } & $\begin{array}{l}\text { Apreciações relativas } \\
\text { à influência da } \\
\text { equoterapia sobre a } \\
\text { psicomotricidade }\end{array}$ \\
\hline 2004 & $\begin{array}{l}\text { Casady, R. } \\
\text { L.; Nicholas- } \\
\text { Larsen, D. S. }\end{array}$ & $\begin{array}{l}\text { The Effect of } \\
\text { Hippotherapy on Ten }\end{array}$ & $\begin{array}{l}\text { No cavalo ocorre adequação } \\
\text { postural mediada pelos } \\
\text { ajustes de feedfoward } \\
\text { e feedback que são } \\
\text { procedimentos de controle } \\
\text { motor, gerados pelo SNC. } \\
\text { Quando controle postural } \\
\text { melhora, as habilidades } \\
\text { motoras também podem } \\
\text { melhorar. }\end{array}$ \\
\hline 2007 & $\begin{array}{l}\text { Silkwood- } \\
\text { Sherer, D. } \\
\text { Warmbier, H. }\end{array}$ & $\begin{array}{l}\text { Effects of Hippotherapy } \\
\text { on Postural Stability, in } \\
\text { Persons }\end{array}$ & $\begin{array}{l}\text { Melhora significativa no } \\
\text { equilibrio dos praticantes. }\end{array}$ \\
& with Multiple Sclerosis: \\
A Pilot Study & & \\
\hline
\end{tabular}




\begin{tabular}{|c|c|c|c|}
\hline 2007 & Benda, W. & $\begin{array}{l}\text { Hippotherapy and the } \\
\text { Significance } \\
\text { of Complementary and } \\
\text { Alternative Medicine }\end{array}$ & $\begin{array}{l}\text { Efeitos do movimento do } \\
\text { cavalo sobre a postura, } \\
\text { equilíbrio e funções gerais. }\end{array}$ \\
\hline 2007 & $\begin{array}{l}\text { Slim, M.; Lebib, } \\
\text { S.; Dziri, C.; } \\
\text { Ben Salah, F. } \\
\text { Z.; Hammadi, } \\
\text { M. }\end{array}$ & $\begin{array}{l}\text { Hippotherapy for } \\
\text { mentally reterded } \\
\text { children - the Tunisian } \\
\text { experience }\end{array}$ & $\begin{array}{l}\text { Benefícios da equoterapia } \\
\text { sobre o equilíbrio, } \\
\text { coordenação motora, } \\
\text { lateralidade, estruturação } \\
\text { espacial e motricidade fina. }\end{array}$ \\
\hline 2007 & Sterba, J. A. & $\begin{array}{l}\text { Does horseback riding } \\
\text { therapy or therapist- } \\
\text { directed hippotherapy } \\
\text { rehabilitate children with } \\
\text { cerebral palsy? }\end{array}$ & $\begin{array}{l}\text { Melhora da função } \\
\text { motora grossa devido aos } \\
\text { mecanismos fisiológicos } \\
\text { relacionados ao movimento } \\
\text { tridimensional do cavalo, } \\
\text { pois os movimentos pélvicos } \\
\text { produzidos são semelhantes } \\
\text { à marcha humana. E, } \\
\text { repostas de endireitamento } \\
\text { e equilíbrio são facilitadas } \\
\text { pelas variações no passo, } \\
\text { velocidade e direção do } \\
\text { cavalo. }\end{array}$ \\
\hline
\end{tabular}




\begin{tabular}{|c|c|c|c|}
\hline 2008 & $\begin{array}{l}\text { Toigo, T.; Leal } \\
\text { Júnior, E. C. P.; } \\
\text { Ávila, S. N. }\end{array}$ & $\begin{array}{l}\text { O uso da equoterapia } \\
\text { como recurso } \\
\text { terapêutico para } \\
\text { melhora do equilíbrio } \\
\text { estático em indivíduos } \\
\text { da terceira idade }\end{array}$ & $\begin{array}{l}\text { Melhora do equilíbrio } \\
\text { estático, a cada passo } \\
\text { do cavalo o centro de } \\
\text { gravidade do praticante } \\
\text { desloca-se da linha média, } \\
\text { o que estimula as reações } \\
\text { de equilíbrio. O sistema } \\
\text { vestibular ao ser solicitado } \\
\text { estimula conexões entre } \\
\text { os canais semicirculares, } \\
\text { onde as oscilações da } \\
\text { endolinfa, provocadas } \\
\text { pelos movimentos da } \\
\text { cabeça (ascendente e } \\
\text { descendente), são captadas } \\
\text { pelas células ciliares e } \\
\text { otólitos. }\end{array}$ \\
\hline 2009 & $\begin{array}{l}\text { Shurtleff, T. L.; } \\
\text { Standeven, J.; } \\
\text { Engsberg, J. R. }\end{array}$ & $\begin{array}{l}\text { Changes in Dynamic } \\
\text { Trunk/Head Stability } \\
\text { and Functional Reach } \\
\text { After Hippotherapy }\end{array}$ & $\begin{array}{l}\text { Melhora no controle } \\
\text { motor do tronco e cabeça, } \\
\text { equilíbrio sentado, assim } \\
\text { demonstrando melhora no } \\
\text { equilíbrio dinâmico. }\end{array}$ \\
\hline 2009 & $\begin{array}{l}\text { McGibbon, N. } \\
\text { H.; Benda, W.; } \\
\text { Duncan, B. } \\
\text { R.; Silkwood- } \\
\text { Sherer, D }\end{array}$ & $\begin{array}{l}\text { Immediate and } \\
\text { Long-Term Effects } \\
\text { of Hippotherapy on } \\
\text { Symmetry of Adductor } \\
\text { Muscle Activity and } \\
\text { Functional Ability in } \\
\text { Children With Spastic } \\
\text { Cerebral Palsy }\end{array}$ & $\begin{array}{l}\text { Promoção de estimulação } \\
\text { vestibular e proprioceptiva, } \\
\text { aumento da consciência } \\
\text { corporal e controle postural, } \\
\text { devido inúmeros estímulos } \\
\text { multissensoriais como os } \\
\text { ajustes posturais que a } \\
\text { criança realiza para manter- } \\
\text { se centralizada sobre o } \\
\text { dorso do cavalo. }\end{array}$ \\
\hline 2010 & $\begin{array}{l}\text { Sanches, } \\
\text { S. M. N.; } \\
\text { Vasconcelos, L. } \\
\text { A. P. }\end{array}$ & $\begin{array}{l}\text { Equoterapia na } \\
\text { reabilitação da } \\
\text { meningoencefalocele: } \\
\text { estudo de caso }\end{array}$ & $\begin{array}{l}\text { Melhora no equilíbrio e } \\
\text { coordenação motora grossa, } \\
\text { gerando influência sobre } \\
\text { movimentos estáticos e } \\
\text { dinâmicos. }\end{array}$ \\
\hline
\end{tabular}




\begin{tabular}{|c|c|c|c|}
\hline 2010 & $\begin{array}{l}\text { Drnach, M.; } \\
\text { O’Brien, P. A.; } \\
\text { Kreger, A. }\end{array}$ & $\begin{array}{l}\text { The Effects of a 5-Week } \\
\text { Therapeutic Horseback } \\
\text { Riding Program on } \\
\text { Gross Motor Function } \\
\text { in a Child with Cerebral } \\
\text { Palsy: A Case Study. }\end{array}$ & $\begin{array}{l}\text { Melhora nas habilidades } \\
\text { motoras grossas, nas } \\
\text { categorias de ficar em } \\
\text { pé, andar, correr e saltar } \\
\text { que exigem adequação } \\
\text { da musculatura postural, } \\
\text { equilíbrio e coordenação. }\end{array}$ \\
\hline 2010 & $\begin{array}{l}\text { Beinotti, F.; } \\
\text { Correia, N.; } \\
\text { Christofoletti, } \\
\text { G.; Borges, G. }\end{array}$ & $\begin{array}{l}\text { Use of hippotherapy } \\
\text { in gait training for } \\
\text { hemiparetic post-stroke }\end{array}$ & Melhora na marcha. \\
\hline 2011 & $\begin{array}{l}\text { Kwon, J.; } \\
\text { Chang, H. J.; } \\
\text { Lee, J. Y.; Ha, } \\
\text { Y.; } \\
\text { Lee, P. K.; Kim, } \\
\text { Y. }\end{array}$ & $\begin{array}{l}\text { Effects of Hippotherapy } \\
\text { on Gait Parameters in } \\
\text { Children With Bilateral } \\
\text { Spastic Cerebral Palsy }\end{array}$ & $\begin{array}{l}\text { Melhora em parâmetros } \\
\text { espaço-temporais } \\
\text { relacionados à velocidade } \\
\text { e tamanho do passo, e } \\
\text { melhora no equilíbrio. }\end{array}$ \\
\hline 2011 & $\begin{array}{l}\text { Zadnikar, M.; } \\
\text { Kastrin, A. }\end{array}$ & $\begin{array}{l}\text { Effects of hippotherapy } \\
\text { and therapeutic } \\
\text { horseback riding on } \\
\text { postural control or } \\
\text { balance in children with } \\
\text { cerebral palsy: a meta- } \\
\text { analysis }\end{array}$ & $\begin{array}{l}\text { Melhora no controle postural } \\
\text { e equilíbrio. }\end{array}$ \\
\hline 2011 & $\begin{array}{l}\text { Frank, A.; } \\
\text { McCloskey, S.; } \\
\text { Dole, R. L. }\end{array}$ & $\begin{array}{l}\text { Effect of Hippotherapy } \\
\text { on Perceived Self- } \\
\text { competence and } \\
\text { Participation in a Child } \\
\text { With Cerebral Palsy }\end{array}$ & $\begin{array}{l}\text { Melhora nas habilidades } \\
\text { motoras grossas, com } \\
\text { permanência sobre o } \\
\text { cavalo em pé, de cócoras e } \\
\text { ajoelhado sem auxílio. }\end{array}$ \\
\hline 2011 & $\begin{array}{l}\text { Granados, A. } \\
\text { C.; Inmaculada } \\
\text { Agı's, I. F. }\end{array}$ & $\begin{array}{l}\text { Why Children With } \\
\text { Special Needs } \\
\text { Feel Better with } \\
\text { Hippotherapy Sessions: } \\
\text { A Conceptual Review }\end{array}$ & $\begin{array}{l}\text { Melhora na coordenação } \\
\text { motora global e equilíbrio. }\end{array}$ \\
\hline
\end{tabular}

Foram encontrados artigos relevantes referentes à temática nas bases pesquisadas a partir de 2004. Desde então, verificou-se o aumento das publicações relacionadas à temática, como podemos visualizar na figura 1: 
Figura 1: Produção anual dos artigos relacionados à temática

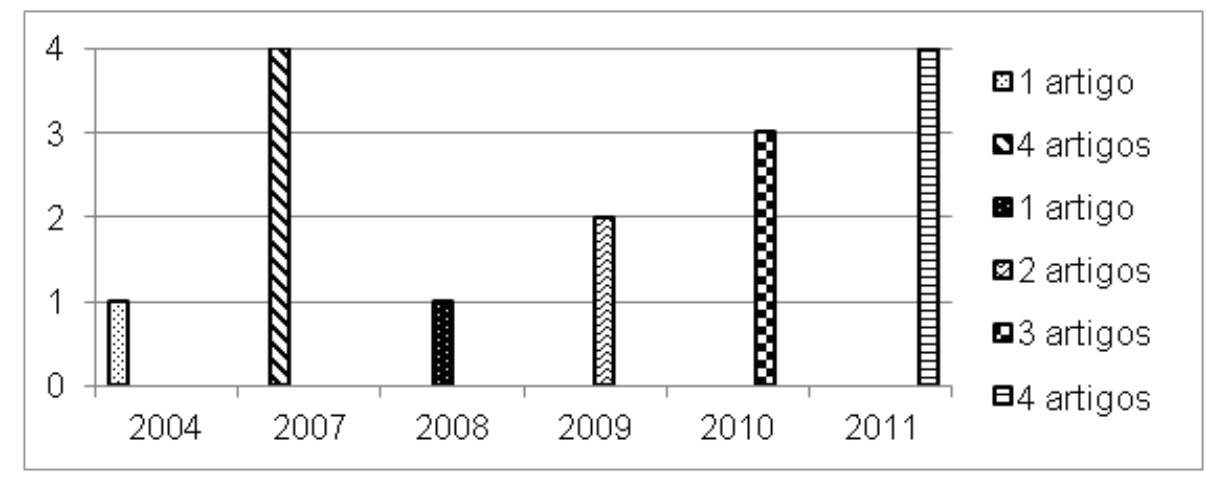

O conhecimento a respeito da influência da equoterapia nos aspectos psicomotores não está sendo abordado de forma direta pela literatura, no entanto, verifica-se pelos achados que a equoterapia promove benefícios e é eficaz quanto às influências sobre os aspectos psicomotores de pessoas com necessidades especiais.

Nota-se que a grande maioria dos estudos equoterápicos é direcionada a pessoas com paralisia cerebral (PC), no entanto, verificou-se que populações diversificadas são beneficiadas pela prática equoterapêutica, como é possível observar na figura 2.

Figura 2: Gráfico referente às populações beneficiadas com a prática equoterapêutica

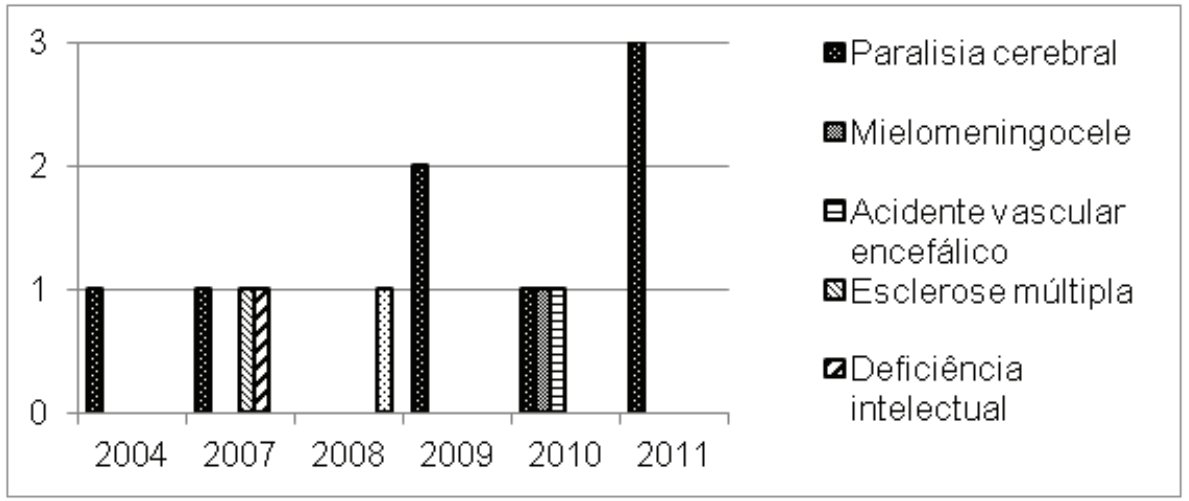

Revista Educação Especial | v. 26 | n. 46 | p. 451-464 | maio/ago. 2013 


\section{Discussão}

Os ganhos na equoterapia ocorrem em decorrência do movimento tridimensional (90 a 110 movimentos por minuto) proporcionado pela andadura ao passo do cavalo, que promove ajuste constante na postura e no equilíbrio, devido à constante aceleração e desaceleração. $O$ cavalo ao passo estimula no praticante, em montaria, movimentação pélvica semelhante ao deslocamento na marcha, podendo auxiliar no equilíbrio dinâmico (SANCHES; VASCONCELOS, 2010; KWON; CHANG; LEE, J.; HA; LEE, P.; KIM, 2011); em termos biomecânicos, o impulso locomotor do cavalo transmitido ao praticante é integrado à força aplicada, do mesmo, para manter o centro de gravidade alinhado durante o deslocamento ao passo (KWON; CHANG; LEE, J.; HA; LEE, P.; KIM, 2011).

Inúmeros são os estímulos proporcionados pela equoterapia, dentre eles o estímulo ao sistema vestibular, que alcança o córtex sensorial e os centros de integração no tronco cerebral e no cerebelo, sendo retransmitido pelo trato corticoespinhal e vias do tronco cerebral até a musculatura periférica, refletindo nas condições de equilíbrio e postura; há também a estimulação proprioceptiva da região cervical que enviam estímulos cervicoespinhais, os quais solicitam e enviam a resposta via reflexo miotático para a musculatura paravertebral extensora e flexora, viabilizando a manutenção da postura (SANCHES; VANCONCELOS, 2010). Além disso, outros estímulos são proporcionados ao praticante em montaria, pois com o posicionamento dos membros inferiores em dorsiflexão, devido ao reflexo de estiramento do gastrocnêmio, em busca de apoio para promover a estabilização há o aprimoramento do controle postural. Padrões apropriados de movimento ocorrem devido à influência do cerebelo na mobilidade, equilíbrio e coordenação (SANCHES; VANCONCELOS, 2010).

O controle postural e equilíbrio são componentes importantes para realização de tarefas motoras, a estabilização postural é essencial para realização de atividades coordenadas e habilidades motoras complexas. Por exemplo, no estudo de Zadnikar e Kastrin (2011) em que abordaram a associação de déficits sensoriais e paralisia cerebral (PC), sugeriram que a equoterapia é uma estratégia que promove melhoras no controle postural e equilíbrio em crianças com PC.

Os resultados encontrados quanto aos benefícios da equoterapia no desenvolvimento psicomotor podem estar embasados em três teorias: a teoria dos sistemas dinâmicos, a de seleção do grupo neuronal e a teoria de integração sensorial (MCGIBBON; HAEHL, 2002; LEWIS, 2000; GRANADOS; AGÍS, 2011). 
A teoria dos sistemas dinâmicos aborda o sistema humano em contínua interação, adaptação e mudança; ocorridas em resposta à dinâmica inter-relação e restrição que ocorrem em três domínios: indivíduo, ambiente e tarefa (LEWIS, 2000). Assim, de acordo com McGibbon e Haehl (2002) os padrões de movimentos resultantes do processo de organização interna envolvem a interação de múltiplas restrições, sendo as mais importantes as relacionadas ao controle postural, motivação e ritmo.

A teoria de seleção do grupo neuronal faz uma relação entre a neurobiologia e o comportamento em três principais categorias: a) a plasticidade neuronal está diretamente relacionada com a evolução da anatomia e a estrutura cerebral, b) a experiência é um dos fatores mais importantes para fixação de resposta correta, no cérebro há diversidade neuronal, porém não é geneticamente determinada, mas modificável com a experiência, c) mapas neuronais são formados através da interação da pessoa com o ambiente, cada pessoa passa por experiências únicas que resultam em conexões neuronais únicas (GRANADOS; AGÍS, 2011). Assim, é possível inferir que por meio dos estímulos multissensoriais da equoterapia e a capacidade de plasticidade neuronal ocorram novas conexões neuronais.

A teoria da integração sensorial, de acordo com Randall apud Granados e Agi's (2011, p. 193), aborda que o comportamento e aprendizagem dependem de uma sucessão de capacidades motoras e sensoriais, onde tais capacidades incluem processamento auditivo, consciência corporal, lateralidade, motricidade fina, planejamento motor, controle visual, percepção do movimento, percepção do toque e percepção viso-espacial. Durante a equoterapia a integração sensorial ocorre quando o cavalo ao passo estimula o sentido tátil, pelo toque e estimulações do ambiente; o sistema vestibular é estimulado pelas mudanças de direção e velocidade, este sistema localizase no interior do ouvido e possui três canais semicirculares preenchidos por endolinfa, tais canais são sensíveis ao movimento; o sistema olfativo responde aos diferentes aromas envolvidos no ambiente hípico e do próprio cavalo; a visão é utilizada para direcionar o cavalo; os muitos sons envolvidos no ambiente hípico auxiliam no desenvolvimento do processamento auditivo. $\mathrm{Na}$ equoterapia todos os sentidos estão trabalhando juntos e de forma integrada, além disso, os proprioceptores (receptores que trazem informação dos músculos, tendões, ligamentos e articulações) estão ativados, resultando em melhora na propriocepção (GRANADOS; AGíS, 2011).

Desse modo, os efeitos proporcionados pela equoterapia são multifatoriais, sendo, portanto a somatória dos ajustes fisiológicos, como mobilização osteoarticular, contração e relaxamento da musculatura agonista e antagonista, e ajustes tônicos e posturais que favorecem respostas 
adaptativas, integram todos os estímulos aferentes e eferentes, que culminam em benefícios psicomotores ao praticante (SANCHES; VASCONCELOS, 2010).

\section{Conclusão}

As teorias do sistema dinâmico, seleção do grupo neuronal e integração sensorial fornecem subsídios para explicar como a equoterapia pode ser uma ferramenta adequada na promoção dos mais diversos estímulos psicomotores, e assim ser eficaz como intervenção voltada a pessoas com diferentes necessidades especiais.

A equoterapia promove benefícios fisiológicos, sociais e educacionais que podem ser verificados em padrões de comportamentos evidenciados em diferentes contextos e populações, como as mencionadas nos estudos, isto é, nos praticantes com paralisia cerebral, mielomeningocele, acidente vascular encefálico, esclerose múltipla, deficiência intelectual e terceira idade.

Mediante ao que foi apresentado, é possível inferir que a equoterapia é uma intervenção que reúne inúmeros estímulos que podem ser ferramentas capazes de influenciar com sucesso diferentes aspectos psicomotores, considerando a possibilidade de desenvolvimento viabilizada pela interação com o cavalo, a movimentação tridimensional e o contato com a natureza. Os ganhos proporcionados pela equoterapia ocorrem devido à plasticidade neuronal, o pico ocorre nos primeiros anos de vida, no entanto, com intervenção adequada é possível modificar e reorganizar o sistema nervoso central, principalmente quando nos referimos à intervenção com a utilização do cavalo, pois há influência em múltiplos sistemas simultaneamente (sensorial, muscular, límbico, vestibular e visual).

\section{Referências}

ANDE-BRASIL. O que é equoterapia. Disponível em: < http://www. equoterapia.org.br/equoterapia.php>. Acesso em: 04 nov. 2011.

BEINOTTI, F.; CORREIA, N.; CHRISTOFOLETTI, G.; BORGES, G. Use of hippotherapy in gait training for hemiparetic post-stroke. Arquivos de neuropsiquiatria, v. 68, n. 6, p. 908-913, 2010.

BENDA, W. Hippotherapy and the significance of complementary and alternative medicine. Alternative \& Complementary Therapies, v. 13, n. 5, p. 266-268, 2007. 
CASADY, R. L.; NICHOLS-LARSEN, D. The effect of hippotherapy on ten children with cerebral palsy. Pediatric Physical Therapy, v. 17, n. 1, p. 165172, 2004.

DRNACH, M.; O'BRIEN, P. A.; KREGER, A. The effects of a 5-week therapeutic horseback riding program on gross motor function in a child with cerebral palsy: a case study. The Journal of Alternative and Complementary Medicine, $v$. 16, n. 9, p. 1003-1006, 2010.

FRANK, A.; MCCLOSKEY, S.; DOLE, R. L. Effect of hippotherapy on perceived self-competence and participation in a child with cerebral palsy. Pediatric Physical Therapy, v. 23, n. 3, p. 301-308, 2011.

GRANADOS, A. C.; AGI'S, I. F. Why children with special needs feel better with hippotherapy sessions: a conceptual rewiew. The Journal of Alternative and Complementary Medicine, v. 17, n. 3, p. 191-197, 2011.

KWON, J.; CHANG, H. J.; LEE. J. Y.; HA, Y.; LEE, P. K.; KIM, Y. Effects of hippotherapy on gait parameters in children with bilateral spastic cerebral palsy. Archives of Physical Medicine and Rehabilitation, v. 92, n. 5, p. 774-779, 2011.

LEWIS, M. D. The promise of dynamic systems approaches for an integrated account of human development. Child Development, v. 71, n. 1, p. 36-43, 2000.

MARCONI, M. A.; LAKATOS, E. M. Técnicas de Pesquisa. São Paulo: Atlas, 1990, p. 56-123.

MCGIBBON, N. H.; BENDA, N. H.; DUNCAN, B. R.; SILKWOOD-SHERER, D. Immediate and long-term effects of hippotherapy on symmetry of adductor muscle activity and functional ability in children with spastic cerebral palsy. Archives of Physical Medicine and Rehabilitation, v. 90, n. 6, p. 966-974. 2009.

MCGIBBON, V.; HAEHL, N. Conceptual Framework for Hippotherapy: is it useful to practice of physical therapy? Disponivel em: <http://www. pediatricapta.org/pass/pubs/CSM\%2002\%20Haehl.ppt>. Acesso em: 04 nov. 2011.

MEREGLIANO, G. Hippotherapy. Physical medicine and rehabilitation clinics of north america, v. 15, n. 4, p. 843-854, 2004. 
SANCHES, S. M. N.; VASCONCELOS, L. A. P.; Equoterapia na reabilitação da meningoendefalocele: estudo de caso. Fisioterapia e Pesquisa, São Paulo, v. 17, n.4, p. 358-361, 2010.

SHURTLEFF, T. L.; STANDEVEN, J. W.; ENGSBERG, J. R. Changes in dynamic trunk/head stability and functional reach after hippotherapy. Archives of Physical Medicine and Rehabilitation, v. 90, n. 7, p. 1185-1195, 2009.

SILKWOOD-SHERES, D.; WRMBIER. H. Effects of hippotherapy on postural stability, in persons with multiple sclerosis: a pilot study. Journal of Neurologic Physical Therapy, v. 31, n. 2, p. 77-84, 2007.

SLIM, M.; LEBIB, S.; DZIRI, C.; BEN SALAH, F. Z.; HAMMADI, M. La thérapie par le cheval dans la réadaptation des enfants handicaps mentaux expérience tunisienne. Journal de Réadaptation Médicale, v. 27, n. 4, p. 115-127, 2007.

STERBA, J. A. Does horseback riding therapy or therapist-directed hippotherapy rehabilitate children with cerebral palsy? Developmental Medicine \& Child Neurology, v. 49, n. 1, p. 68-73, 2007.

TOIGO, T.; LEAL JÚNIOR, E. C. P.; ÁVILA, S. N. O uso da equoterapia como recurso terapêutico para melhora do equilíbrio estático em indivíduos da terceira idade. Revista Brasileira de Geriatria e Gerontologia, v. 11, n. 3, p. 391-403, 2008.

WICKERT, H. O cavalo como instrumento cinesioterapêutico. Disponível em: < http://www.equoterapia.org.br/trabalho_ver.php?indice=104>. Acesso em: 04 nov. 2011.

ZADNIKAR, M.; KASTRIN, A. Effects of hippotherapy and therapeutic horseback riding on postural control or balance in children with cerebral palsy: a meta-analysis. Developmental Medicine \& Child neurology, v. 53, n. 8, p. 684-691, 2011.

\section{Notas}

${ }^{1}$ Um ou mais membros em constante contato com o chão.

2 Movimentos que ocorrem de um lado do animal, ocorrem igual e simetricamente do outro.

${ }^{3}$ Cadenciada a quatro tempos - entre o elevar e o pousar de um membro quatro batidas distintas são ouvidas. 
Gardenia de Oliveira Barbosa - Mey de Abreu van Munster

\section{Correspondência}

Gardenia de Oliveira Barbosa - Rua Prof. José Ferraz de Camargo, 375, Apto 08. CEP: 13.566-440 - Vila Celina; São Carlos - São Paulo.

E-mail: garativ@gmail.com - mey@ufscar.br

Recebido em 27 de fevereiro de 2012

Aprovado em 13 de julho de 2012 